\title{
Álcool E Direção: Uma Realidade Entre Os Estudantes
}

\author{
${ }^{1}$ Vanessa Brito Miguel Couto, ${ }^{2}$ Bernardo Pires Sampaio, ${ }^{3}$ Leonardo de Lima Rosas, ${ }^{4}$ Ana Carla de Santana Costa \\ ${ }^{5}$ Ana Lúcia de Camargo Corrêa, ${ }^{6}$ Kadja de Morais Correia K, ${ }^{7}$ Ravel Souza Alves, ${ }^{8}$ Gabriela Santos Silva \\ ${ }^{9}$ Roberto Almeida Rego de Souza, ${ }^{10}$ Irany Santana Salomão
}

\section{RESUMO}

Histórico: Os índices de acidentes de trânsito causados por motoristas alcoolizado conservam-se altos, principalmente quando se considera a população de estudantes, na qual o consumo de álcool é maior que na população em geral. Assim, este estudo tem como objetivo identificar a prevalência do binômio bebida alcóolica e direção em estudantes no município de Ilhéus, Brasil.

Desenho de Estudo: Trata-se de um trabalho prospectivo e quantitativo em que foi aplicado um questionário aleatoriamente em 192 estudantes que se encontravam em bares na cidade de Ilhéus durante o mês de maio de 2016.

Resultados: O sexo masculino foi discretamente mais prevalente $(53,1 \%)$, com predominância da faixa etária entre 20 e 30 anos $(71,9 \%)$ e daqueles que cursavam o ensino superior $(70,3 \%)$. Os resultados da pesquisa apontaram que $76,5 \%$ beberam pela primeira vez entre 12 e 17 anos; $50 \%$ experimentaram bebida alcóolica numa festa ou evento social e $43,7 \%$ começaram a ingerir álcool por causa dos amigos. Quanto ao hábito de dirigir após consumir bebida alcóolica, $62,5 \%$ relataram possuí-lo e a maioria dos entrevistados pegariam carona sabendo que o motorista ingeriu álcool $(78,1 \%)$. Apesar disso, quase todos os estudantes $(97 \%)$ consideram-se bem informados quanto à problemática do consumo de álcool, principalmente ao associá-lo com a direção.

Conclusão: A maioria dos jovens universitários costuma ingerir bebida alcóolica e dirigir. Além disso, ignoram o fato do motorista estar alcoolizado ao aceitarem uma carona, apesar de terem consciência dos prejuízos advindos dessa associação.

Palavras-chave: Bebidas alcoólicas, Condução de veículo, Estudantes.

How to cite this article: Couto VBM, Sampaio BP, de Lima Rosas L, de Santana Costa AC, de Camargo Corrêa AL, de Morais Correia K, Alves RS, Silva GS, de Souza RAR, Salomão IS. Álcool EDireção: Uma Realidade Entre Os Estudantes. Panam J Trauma Crit Care Emerg Surg 2017;6(2):68-71.

Source of support: Nil

Conflict of interest: None

\footnotetext{
${ }^{1-9}$ Estudante, ${ }^{10}$ Mestre

${ }^{1-10}$ Departamento de Ciências da Saúde, Universidade Estadual de Santa Cruz, Ilhéus, Bahia, Brazil

Corresponding Author: Vanessa Brito Miguel Couto, Estudante Departamento de Ciências da Saúde, Universidade Estadual de Santa Cruz, Ilhéus, Bahia, Brazil, Phone: +(73)32313924 e-mail: nessa_couto@hotmail.com
}

\section{ABSTRACT}

Background: The rates of traffic accidents caused by alcoholic drivers remain high, especially when considering the student population, in which alcohol consumption is higher than in the general population. Thus, this study aims to identify the prevalence of the alcoholic beverage and driving binomial in students in the city of Ilheus, Brazil.

Study design: It is a prospective and quantitative study in which a questionnaire was randomly applied to 192 students who were in bars in the city of llhéus during the month of May 2016.

Results: The male gender was slightly more prevalente (53.1\%), with a predominance of the age group between 20 and 30 years $(71.9 \%)$ and those who were enrolled in college (70.3\%). The results of the research indicated that $76.5 \%$ drank for the first time between 12 and 17 years; 50\% experienced alcoholic drink at a party or social event and $43.7 \%$ started drinking alcohol because of friends. As for the habit of driving after consuming alcohol beverages, $62.5 \%$ reported owning it and most of the interviewees would take a ride knowing that the driver ingested alcohol (78.1\%). Nevertheless, almost all students (97\%) consider themselves well informed about the problematic alcohol consumption, especially when associating it with driving.

Conclusion: The majority of college students usually drink alcohol and drive. In addition, they ignore the fact that the driver is drunk when accepting a ride, despite being aware of the damages caused by this association.

Keywords: Alcoholic Beverages, Automobile Driving, Students.

\section{INTRODUÇÃO}

O consumo de álcool constitui um problema mundial, levando a altas taxas de mortalidade anualmente, principalmente entre os jovens. ${ }^{1}$ Essa substância gera consequências tanto físicas como psicológicas, problemas relacionais, agressividade, acidentes domésticos, do trabalho e de trânsito, comportamento sexual de risco, absentismo escolar e agravos, como acidente vascular encefálico e câncer. ${ }^{2-4}$

O consumo de álcool pela comunidade universitária é mais frequente do que na população em geral e seu uso abusivo tem alcançado estudantes dos mais diversos graus educacionais. ${ }^{3,5}$ São diversos os motivos pelos quais os estudantes apresentam ingestão excessiva de bebidas alcoólicas, pode-se destacar as causas emocionais e sociais, por exemplo, o uso como estimulador emocional, como facilitador social, como mecanismo de fuga dos sentimentos negativos ou para simples alívio do estresse cotidiano ${ }^{3,4}$. 
O sentimento de invulnerabilidade e onipotência característico dos jovens, quando aliado aos efeitos do álcool acabam por culminar em altas taxas de acidentes automobilísticos. ${ }^{6}$ Os índices de acidentes de trânsito causados por motoristas alcoolizados ainda são altos em todo o mundo, ${ }^{7}$ devido a isso o Brasil aprovou duas leis federais proibindo o consumo de álcool e direção: a Lei 11.705, de 2008, e a Lei Seca - Lei 12.760 - com o propósito de assegurar e qualificar a eficácia do motorista com a intenção de minimizar os acidentes de trânsito e preservar a vida do cidadão. ${ }^{8,9}$ Segundo um estudo realizado por Malta e colaborados ${ }^{8}$ utilizando dados do Vigitel, essa estratégia resultou em uma redução de $45 \%$ na prevalência de consumo abusivo de álcool e direção na população adulta, porém ocorreu de forma desigual nas diferentes regiões do país, evidenciandose pequena repercussão nas taxas de mortalidade por acidentes de transporte terrestre dos estados do Norte, Centro-oeste e Nordeste, levantando-se a hipótese de menor fiscalização nesses locais. ${ }^{10}$ Diante disso, neste trabalho objetiva-se identificar a prevalência do binômio bebida alcóolica e direção em estudantes no município de Ilhéus.

\section{MÉTODOS}

Trata-se de um trabalho prospectivo de caráter quantitativo e descritivo realizado com 192 estudantes que encontravam-se em bares selecionados aleatoriamente na cidade de Ilhéus-BA durante o mês de maio de 2016. Determinou-se como critério de inclusão a profissão de estudante e como critério de exclusão a total abstinência de bebidas alcoólicas. Para a escolha dos entrevistados, abordou-se todos os presentes nos bares nos períodos de coleta de dados, explicando-os sobre o estudo e questionando-lhes a profissão.

A técnica de coleta de dados foi a aplicação de questionários com perguntas objetivas abordando as seguintes variáveis: sexo; idade; etnia; estado civil; grau de escolaridade; início do consumo de bebidas alcoólicas (idade, local e motivo); ambientes e companhias habituais para ingestão de álcool; presença do binômio álcool e direção; presença da atitude de aceitar carona de motoristas alcoolizados; consumo de bebida alcoólica no dia da entrevista e posterior condução de automóvel; informação acerca dos problemas relacionados ao álcool e deste com a direção; envolvimento em acidentes de trânsito devido à presença de alcoolemia.

Os participantes foram esclarecidos sobre objetivos, sigilo e anonimato e foi-lhes permitido tomar a decisão de participar da pesquisa ou não. Os entrevistadores foram previamente treinados quanto a aplicação do questionário e realizou-se um momento de pré-teste para avaliação da uniformidade da mesma. Após a coleta de dados, procedeu-se à etapa de análise dos dados por meio de estatística descritiva simples, resultando na construção de tabelas e gráficos, para melhor visualização e entendimentos dos resultados.

\section{RESULTADOS}

O sexo masculino foi discretamente mais prevalente $(53,1 \%)$. As faixas etárias se distribuíram da seguinte forma: abaixo de 20 anos $(17,1 \%)$, entre 20 e 30 anos $(71,9 \%)$ e acima de 30 anos (11\%), com idade média igual a 24,5 anos. Os entrevistados se autodeclararam caucasianos em $40,6 \%$ dos casos, pardos em $37,5 \%$ e negros em $21,9 \%$. Quanto ao estado civil, $90,6 \%$ eram solteiros. A grande maioria dos estudantes $(70,3 \%)$ eram universitários e 9,4\% cursavam o ensino médio.

Em relação à idade início do consumo de bebidas alcóolicas, 76,5\% beberam pela primeira vez entre $12 \mathrm{e}$ 17 anos e $12,5 \%$ após os 18 anos, sendo que $50 \%$ experimentaram bebida alcóolica numa festa ou evento social, $25 \%$ em casa sozinhos ou com amigos e 15,6\% em casa acompanhados da família. No que concerne o motivo da iniciação ao consumo de álcool, 43,7\% começaram a ingerir bebidas alcoólicas por causa dos amigos, $39 \%$ por curiosidade e 7,8\% devido ao incentivo de familiares.

A maioria afirmou que costuma beber em festas ou em bares $(89 \%)$, sendo que apenas $9,4 \%$ elegeram a própria residência como local habitual do consumo de bebidas alcoólicas. Uma grande parcela dos entrevistados referiu que costuma ingerir álcool quando acompanhados pelos amigos $(87,5 \%)$ e uma minoria $(3,1 \%)$ relatou o costume de beber sozinho.

Quanto ao hábito de dirigir após consumir bebida alcóolica, $62,5 \%$ relataram possuí-lo, sendo que destes $67,5 \%$ eram do sexo masculino e $82,5 \%$ estavam entre 20 e 30 anos de idade. A maioria dos entrevistados pegariam carona sabendo que o motorista ingeriu álcool (78,1\%). Em relação ao dia em que foram realizadas as entrevistas, $70,3 \%$ estavam consumindo bebidas alcoólicas, sendo que destes 22,2\% iriam dirigir ao deixar o local. Apesar disso, quase todos os estudantes (97\%) consideravamse bem informados quanto à problemática do consumo de álcool, principalmente ao associá-lo com a direção. Do total da amostra, $14 \%$ referiram já ter se envolvido em um acidente automobilístico devido o consumo de bebida alcóolica.

\section{DISCUSSÃO}

Os resultados apontaram que a diferença entre os sexos quanto ao consumo de álcool foi demasiadamente pequena corroborando com Barbosa e colaboradores ${ }^{11}$, isso denota uma elevada ingestão de bebidas alcoólicas pelo sexo feminino, sendo um motivo de preocupação, 
já que a literatura documenta maior susceptibilidade desse gênero aos efeitos lesivos do álcool. ${ }^{11}$ Em relação a idade, o estudo demonstra um predomínio do consumo de bebidas alcoólicas em adultos jovens, em concordância com os dados contidos na literatura. ${ }^{12,13}$ Quanto ao estado civil, a grande maioria dos estudantes alcoolistas são solteiros, corroborando com outros estudos. ${ }^{11-13}$ No que se refere ao grau de escolaridade, a maioria dos entrevistados eram universitários, sendo que a literatura destaca os altos índices de consumo de bebidas alcoólicas em estudantes no ensino superior. ${ }^{14-16}$

A maioria dos estudantes iniciam o consumo de bebidas alcoólicas antes da idade permitida por lei para a venda dessas substâncias, corroborando com a literatura. ${ }^{17,18}$ Percebeu-se que a introdução à ingestão de álcool se deu principalmente em eventos sociais acompanhados por amigos e tendo como motivo principal a influência destes, seguido pela curiosidade, fato que pôde ser concluído por Chiapetti e Serbena, ${ }^{19}$ apesar de ir de encontro a Stamm e Bressam ${ }^{17}$ que apontaram a presença da família como ambiente do primeiro contato com bebida alcoólica.

Em relação aos locais habituais de consumo alcoólico, destacou-se festas e bares assim como em um estudo realizado com estudantes da Famed/UFU ${ }^{18}$ no qual $72,45 \%$ da amostra tinha como situação propícia à ingestão de álcool as festas de faculdade. No que se refere a companhia frequente para o uso de bebidas alcoólicas, constatou-se que a maioria dos entrevistados o fazem na presença de amigos, sendo mínima a porcentagem daqueles que consomem habitualmente sozinhos, assim como demonstrado em uma pesquisa realizada em uma universidade de Curitiba ${ }^{19}$.

No que concerne o hábito de beber e dirigir, o presente estudo identificou que a maioria dos estudantes o possui, em contraposição a duas outras pesquisas realizadas em Belo Horizonte e Diadema, ${ }^{20,21}$ nas quais a maioria dos entrevistados referiram não costumar dirigir após o consumo de bebida, apesar de terem bebido e estarem conduzindo automóvel no dia em que foram submetidos aos questionários, porém ressalta-se que em ambas, no momento em que realizou-se o estudo estavam presentes forças policiais acompanhando os pesquisadores ao contrário deste. Em relação ao perfil do indivíduo que refere ingerir bebida alcoólica e posteriormente dirigir, houve predomínio do sexo masculino e da faixa etária de 20 a 30 anos de idade, assim como descrito na literatura. . $^{21,22}$

Apesar das altas taxas de transgressão a Lei Seca identificadas, quase a totalidade dos estudantes relataram ter consciência dos problemas relacionados ao consumo de bebidas alcoólicas, principalmente no que se refere a sua associação com a direção corroborando com o estudo realizado por Campos e colaborado ${ }^{20}$ e contrariando as recomendações realizados por Stamm e Bressam ${ }^{17}$ que aventam a necessidade de desenvolver uma conscientização a respeito das consequências do álcool para prevenir seus efeito nocivos, já que percebeu-se que apesar da mesma existir nessa amostra, não coibi a combinação álcool e direção.

Uma parcela significativa, cerca de 1 em cada 7 entrevistados, referiram já ter se envolvido em algum momento de sua vida em acidentes automobilísticos em decorrência dos efeitos do álcool em concordância com diversos estudos da literatura que destacam a presença de alcoolemia como um fator frequente nas vítimas de acidentes de trânsito. ${ }^{23-26}$

Dessa forma, conclui-se que os jovens universitários predominaram entre estudantes entrevistados nos bares, sendo que a maioria iniciou precocemente o consumo de bebidas alcoólicas principalmente devido à influência dos amigos. Além disso, ainda hoje, a maior parte deles costuma associar álcool e direção e ignorar o fato do motorista estar alcoolizado ao aceitarem uma carona, apesar de terem consciência dos prejuízos advindos dessa associação. Por isso, os autores deste estudo sugerem que a melhor maneira de reduzir os índices do binômio álcool e direção seria uma maior fiscalização do trânsito, de acordo com as leis federais vigentes, e punição por meio de multas àqueles os quais as descumprem, isso porque, Ilhéus encontrar-se deficiente nessas ações de prevenção de acidentes automobilísticos. Ademais, essa sugestão é sustentada pelo fato de a fiscalização ter sido descrita como estratégia de maior impacto no comportamento do condutor quando comparada à educação e à engenharia em uma metanálise realizada por Aguilera, Moysés e Moysés. ${ }^{27}$

\section{REFERÊNCIAS}

1. Imai FI, Coelho IZ, Bastos JL. Consumo excessivo de álcool, tabagismo e fatores associados em amostra representativa de graduandos da Universidade Federal de Santa Catarina, 2012: estudo transversal. Epidemiol Serv Saúde 2014;23(3):435-446.

2. Cardoso FM, Barbosa HA, da Costa FM, Vieira MA, Caldeira AP. Fatores associados à prática do binge drinking entre estudantes da área de saúde. Rev CEFAC 2015;17(2):475-484.

3. Soares WD, Jones KM, Finelli LAC, Araujo TP, Barros KSJ. Álcool como mediador social em universitários. Rev Bras Promoç Saúde 2015;28(3):427-433.

4. Rodrigues PFS, Salvador ACF, Lourenço IC, Santos LR. Padrões de consumo de álcool em estudantes da Universidade de Aveiro: relação com comportamentos de risco e stress. Análise Psicológica 2014;32(4):453-466.

5. Costa SB. Perfil do consumo de álcool e tabaco entre universitários dos cursos de humanas de uma universidade pública. Trabalho de Conclusão de Curso (Graduação em Enfermagem) - Universidade Estadual da Paraíba, Centro de Ciências Biológicas e da Saúde, 2014.

6. Rodriguez CF; Kovács MJ. O que os jovens têm a dizer sobre as altas taxas de mortalidade na adolescência? Imaginario 2005;11(11):111-136.

7. Almeida ND, Roazzi A. Álcool e direção em universitários, comunicação persuasiva e prevenção. Psicologia: Ciência e Profissão 2014;34(3):715-732. 
8. Malta, DC, Berna RTI, da Silva MMA, Claro RM, da Silva Júnior JB, dos Reis AAC. Consumo de bebidas alcoólicas e direção de veículos, balanço da lei seca, Brasil 2007 a 2013. Rev Saúde Pública 2014;48(4):692-696.

9. Da Silva, MLL. Álcool versus direção automobilística: um caminhar separados. Revista Tropos 2015;1(4):1-14.

10. Malta DC, Filho AMS, Montenegro MMS, Mascarenhas MDM, da Silva MMA, Lima CM, Neto OLM, Temporão JG, Penna GO. Análise da mortalidade por acidentes de transporte terrestre antes e após a Lei Seca - Brasil, 2007-2009. Epidemiol Serv Saúde 2010;19(4):317-328.

11. Barbosa FL,Barbosa RL, Barbosa MCL, de Aguiar DL, Figueiredo IA, Ribeiro AC, de Castro ITC, . Uso de álcool entre estudantes de Medicina da Universidade Federal do Maranhão. Rev Brasil Educ Méd 2013;37(1):89-95.

12. Fachin A, Furtado EF. Uso de álcool e expectativas do beber entre universitários: Uma análise das diferenças entre os sexos. Psic Teor Pesq 2013;29(4):421-428.

13. Da Silva L, Vieira NA, Falavigna MF. A incidência do consumo de álcool entre graduandos do curso de administração de uma instituição de ensino superior privada do Médio Vale do Paraíba. REENVAP Lorena 2015;1(8):11-28.

14. Silva JN, Rodrigues MG, Jones KM, Finelli LAC, Soares WD. Consumo de álcool entre universitários. RBPeCS 2015;2(2): $35-40$.

15. Baumgarten LZ, Gomes VLO, Da Fonseca AD. Consumo alcoólico entre universitários (as) da área da saúde da Universidade Federal do Rio Grande do Sul/RS: Subsídios para enfermagem. Esc Anna Nery 2012;16(3):530-535.

16. Ramis TR, Mielke GI, Habeyche EC, Oliz MM, Azevedo MR, Hallal PC. Tabagismo e consumo de álcool em estudantes universitários: prevalência e fatores associados. Rev Bras Epidemiol 2012;15(2):376-385.

17. Stamm M, Bressan L. Consumo de álcool entre estudantes do curso de enfermagem de um município do oeste catarinense. Cienc Cuid Saude 2007;6(3):319-324.
18. PaduaniGF,BarbosaGA,deMoraisJCR,PereiraJCP,AlmeidaMF, Prado MM, de Almeida NBC, Ribeiro MA. Consumo de álcool e fumo entre os estudantes da Faculdade de Medicina da Universidade Federal de Uberlândia. Rev Bras Educ Méd 2008;32(1):66-75.

19. Chiapetti N, Serbena CA. Uso de álcool, tabaco e drogas por estudantes da área de saúde de uma universidade de Curitiba. Psicol: Reflex Crít 2007;20(2):303-313.

20. Campos VR, Salgado R, Rocha MC, Duailibi S, Laranjeira R. Prevalência do beber e dirigir em Belo Horizonte, Minas Gerais, Brasil. Cad Saúde Pública 2008;24(4):829-834.

21. Duailibi S, Pinsky I, Laranjeira R. Prevalência do beber e dirigir em Diadema, estado de São Paulo. Rev Saúde Pública 2007;41(5):1-4.

22. Moura EC, Malta DC, Neto OLM, Penna GO, Temporão JG. Direção de veículos motorizados após consumo abusivo de bebidas alcoólicas, Brasil, 2006 a 2009. Rev Saúde Pública 2009;43(5):891-894.

23. Abreu AMM, De Lima JMB, Griep RH. Acidentes de trânsito e a frequência dos exames de alcoolemia com vítimas fatais na cidade do Rio de Janeiro. Esc Anna Nery Rev Enferm 2009;13(1):44-50.

24. Abreu AMM, de Lima JMB, Matos LN, Pillon SC. Uso de álcool em vítimas de acidentes de trânsito: estudo do nível de alcoolemia. Rev Latino-Am Enfermagem 2010;18(Spe): 513-520.

25. Abreu AMM, De Lima JMB, Alves TR. O impacto do álcool na mortalidade em acidentes de trânsito: uma questão de saúde pública. Esc Anna Nery R Enferm 2006;10(1): 87-94.

26. Marín-León L, Vizzotto MM. Comportamentos no trânsito: um estudo epidemiológico com estudantes universitários. Cad Saúde Pública 2003;19(2):515-523.

27. Aguilera SLVU, Moysés ST, Moysés SJ. Intervenções de segurança viária e seus efeitos nas lesões causadas pelo trânsito: uma revisão sistemática. Rev Panam Salud Publica 2014;36(4):257-265. 